\title{
ELECTRO-OPTIC EFFECT IN STRAINED AND LATTICE MATCHED MULTIQUANTUM WELL STRUCTURES - ROLE OF EXCITONIC RESONANCES
}

\author{
J. Pamulapatia , J. P. Loehr ${ }^{a}$, J. Singh ${ }^{a}$, P. K. Bhattacharya ${ }^{a}$, and \\ M. J. Ludowise ${ }^{b}$ \\ ${ }^{a}$ Department of Electrical Engineering and Computer Science \\ The University of Michigan \\ Ann Arbor, MI 48109-2122 USA \\ ${ }^{b}$ Hewlett-Packard Laboratories \\ 3500 Deer Creek Road \\ Palo Alto, CA 94304 USA
}

(Received 30 July 1990)

\begin{abstract}
The well known quantum confined Stark effect (QCSE) is currently being exploited to design optoelectronic devices based on electric field controlled absorption of photons. QCSE is also responsible for strong below exciton resonance changes in refractive index with applied field. These changes can be used for high speed couplers and switches in waveguides. This paper focuses on the use of strain to influence the excitonic resonances in $\mathrm{In}_{x} \mathrm{Ga}_{1-x} \mathrm{As} / \mathrm{Al}_{0.2} \mathrm{Ga}_{0.8} \mathrm{As}$ and $\mathrm{In}_{0.53 \pm x} \mathrm{Ga}_{0.47 \mp x} \mathrm{As} / \mathrm{InGaAsP}$ multiple quantum wells. Experimental results are presented showing the effect of compressive and tensile strain on the refractive index changes. Theoretical results including the important $\mathrm{HH}-\mathrm{LH}$ band mixing effects are presented for the electrooptic effect. In particular, the contribution of the excitonic part is discussed and is found to dominate the electro-optic effect near the bandedge.
\end{abstract}

\section{Introduction}

The electro-optic properties of quantum wells are very different from that of single layered materials. In a quantum well the valence band degeneracy is removed and this causes changes in the optical absorption. With the application of a transverse electric field there are changes in the energy eigenvalues of the quantum well and the coupling of electron and holes in the subband with the same quantum number are reduced. Theoretically, it has been shown, by considering only bandto-band transitions, that there is a large refractive index change for the $\mathrm{TE}$ mode in a multiple quantum well (MQW) structure, resulting in a large electro-optic coefficient. ${ }^{1}$ Experimentally, it has been observed that the quadratic electro-optic effect becomes dominant as one approaches the excitonic edge. ${ }^{2}$

Biaxial strain in quantum wells changes the energy separation and coupling between the heavy hole ( $\mathrm{HH})$ and light hole (LH) excitonic transitions. In the case of compressive strain, the $\mathrm{HH}$ absorption dominates the absorption edge, while in the case of tensile strain the light hole absorption dominates. Since the TE mode couples to the HH while the TM mode couples to the $\mathrm{LH}$, this HH - LH splitting is expected to play an important role in determining the electro-optic properties. The effect of biaxial strain on the electro-optic effect in quantum wells have not been investigated in detail, both theoretically and experimentally, other than some preliminary experiments by us ${ }^{3}$ on $\mathrm{InGaAs}_{\mathrm{GaAs}} \mathrm{MQW}$.

This paper focuses on the use of strain to influence the excitonic resonances. Experimental results are presented showing the effect of compressive and tensile strain on the refractive index changes. Measurements are carried out on molecular beam epitaxial (MBE) $\mathrm{In}_{x} \mathrm{Ga}_{1_{-x}} \mathrm{As} / \mathrm{Al}_{0.2} \mathrm{Ga}_{0.8} \mathrm{As}$ MQW structures for compressive strain and metallorganic chemical vapor deposition (MOCVD) $\operatorname{In}_{0.53 \pm x} \mathrm{Ga}_{0.47 \mp x} \mathrm{As} / \mathrm{InGaAsP}$ structures for compressive and tensile strain. The phase shift is measured as a function of applied bias in waveguide electro- 
optic devices. Far from the excitonic resonances the $\Delta \mathrm{n}$ vs. E-ficld relation is linear, but it develops a quadratic behavior as one approaches the exciton peak. Theoretical results including the important HH-LH band mixing effects are presented for the electro-optic effect. We calculate the absorption coefficient directly from the computed bandstructure and compute the associated refractive index change by using the Kramers-Kronig transform. In particular, the contribution of the excitonic part is discussed. The data is the first ever reported (i) detailing the effects of strain on the electro-optic coefficients and (ii) calculating the effect of strain on the electro-optic coefficients with the inclusion of excitons.

\section{Theory}

To model the optical properties of semiconductor quantum wells it is important to include the strong coupling between the heavy hole $\left(\mathrm{HH},\left|\frac{3}{2}, \pm \frac{3}{2}\right\rangle\right)$, and light hole ( $\left.\mathrm{LH},\left|\frac{3}{2}, \pm \frac{1}{2}\right\rangle\right)$ states; the conduction band may be treated in the parabolic approximation. We account for the valence band coupling by using the $4 \times 4 \mathrm{k} \cdot \mathrm{p}$ Hamiltonian. ${ }^{4}$ The effect of strain is incorporated by including a splitting $\delta$ between the light and heavy hole diagonal elements. For $\operatorname{In}_{x} \mathrm{Ga}_{1-x}$ As grown on $\mathrm{GaAs}$ and $\mathrm{In}_{0.53+x} \mathrm{Ga}_{0.47-x}$ As grown on InP it is given (in $\mathrm{eV}$ ) by $\delta=-5.966 \epsilon$, where the lattice mismatch $\epsilon$ is related to

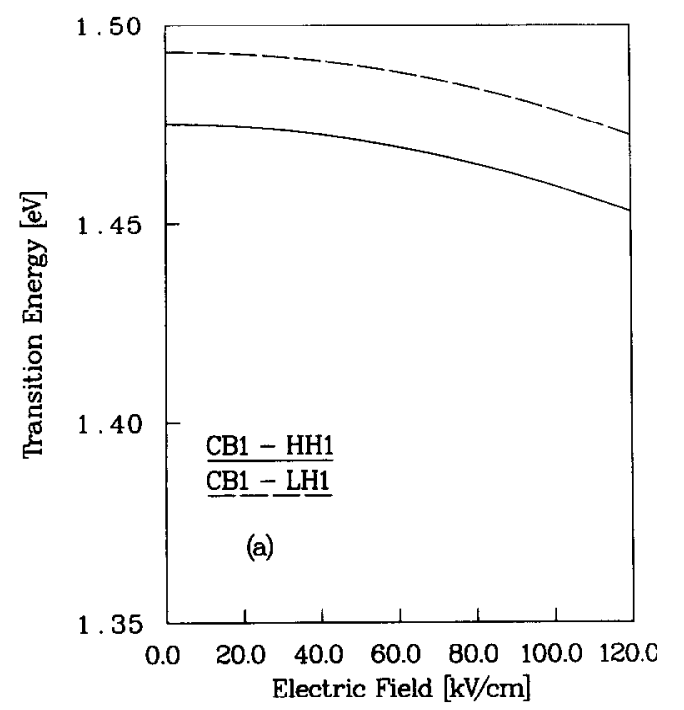

the excess In composition $x$ by $\epsilon=-0.07 x .^{5}$ This splitting reduces the off-diagonal mixing between the $\mathrm{HH}$ and LH states and changes the effective masses of the holes. In addition, the strain-induced splitting dramatically changes the difference between the light and heavy hole subband energies. We solve the Hamiltonian in the presence of strain and electric fields by using a finite difference numerical technique. ${ }^{6}$ We also solve for the electron states in the presence of fields and compute the transition energies shown in Fig. 1. We see that the electric field shifts both the light and heavy hole transition energies quadratically (the quantum confined Stark effect). In addition, we see that the strain strongly affects the zero-field transition energies for heavy and light holes. In the lattice matched results of Fig. 1(a) the light hole bandgap is slightly larger than that of the heavy hole because of the larger quantization energy associated with its lighter mass. The application of compressive strain, though, greatly increases this separation; applying tensile strain reduces the light hole energy below that of the heavy hole. The splitting strongly influences the optical properties because of the polarization dependence of the selection rules applying to optical transitions. TE mode ( $\hat{x}$-polarized) light couples three times more strongly to HH states than to LH states and TM mode ( $\hat{z}$-polarized) light couples only to the LH states; ${ }^{7}$ growth is done in the $z$-direction. Hence, the

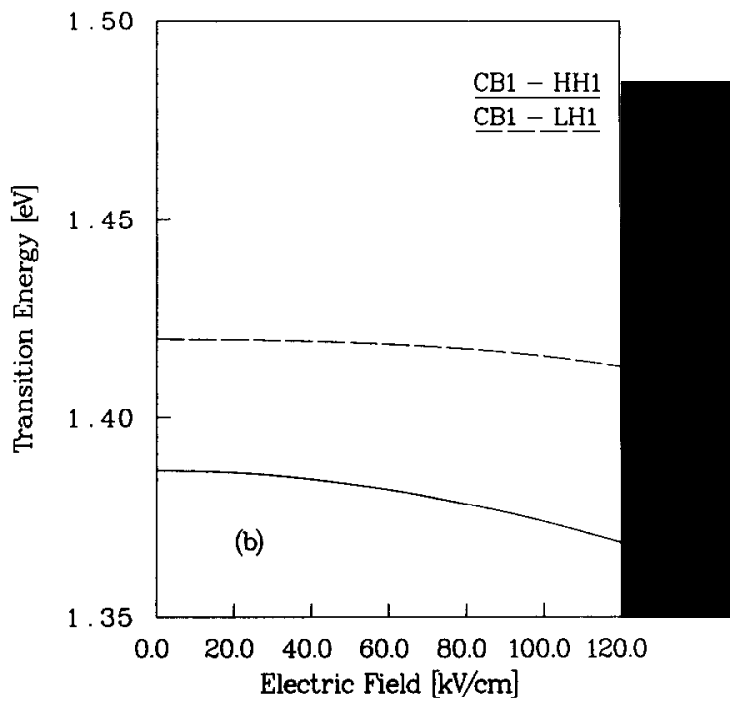

Figure 1: Transition energies between the lowest conduction subband (CB1) and the first heavy hole (HH1) and light hole (LH1) subbands for a $75 \AA \mathrm{In}_{x} \mathrm{Ga}_{1-x} \mathrm{As} / \mathrm{Al}_{0.2} \mathrm{Ga}_{0.8} \mathrm{As}$ (on GaAs) quantum well; $x=0.00$ in (a), $x=0.09$ in (b). 
separation between $\mathrm{HH}$ and $\mathrm{LH}$ states will strongly affect the absorption spectra and the associatcd bircfringence. After obtaining the bandstructure we calculate the excitonic binding energies and oscillator strengths via a nonvariational numerical technique. ${ }^{8}$

To determine the effect of strain and electric fields on the refractive index, we apply the Kramers-Kronig transform to the absorption spectra, yielding the result

$$
n\left(\omega_{0}\right)-1=\frac{c}{\pi} P \int_{0}^{\infty} \frac{\alpha(\omega) d \omega}{\omega^{2}-\omega_{0}^{2}} .
$$

Since we expect excitonic effects to dominate, we include only the excitonic contribution to the absorption. It has been observed experimentally ${ }^{9}$ that the total area under the absorption curve (for energies slightly above the bandgap) remains constant with the application of electric field. This reflects the sum rules that hold for excitonic and band to band absorption: as the electric field breaks the reflection symmetry of the potential, the parity-allowed transitions have their absorption strengths decreased and the parity-forbidden transitions begin to pick up, causing the total area to remain approximately constant. Since a calculation of all the ground and excited state excitons formed between all subbands as a function of strain and electric field would be computationally cumbersome, we include only the CB1-HH1 and CB1-LH1 excitons in our calculated absorption spectra. In order to conserve area in the presence of electric fields, we keep the oscillator strengths of these excitons constant as a function of electric field; this approximation is justified by the agreement obtained with the experimental trends. We assume the excitonic broadening to be by a Lorentzian function with half-width $\Gamma$

$$
\Delta\left(\omega \quad \omega^{\prime}\right)=\frac{\Gamma}{\pi\left[\left(\omega-\omega^{\prime}\right)^{2}+\Gamma^{2}\right]}
$$

and perform the Kramers-Kronig transform by using the result

$$
P \int_{0}^{\infty} d \omega \frac{\Delta\left(\omega-\omega^{\prime}\right)}{\omega^{2}-\omega_{0}^{2}}=\frac{-\left[\omega_{0}^{2}-\left(\omega^{\prime}\right)^{2}+\Gamma^{2}\right]}{\left[\omega_{0}^{2}+\left(\omega^{\prime}\right)^{2}+\Gamma^{2}\right]^{2}-4 \omega_{0}^{2}\left(\omega^{\prime}\right)^{2}}
$$

obtained by contour integration.

\section{Experimental Techniques}

Experiments were performed with $\mathrm{In}_{x} \mathrm{Ga}_{1-x}$ As/ $\mathrm{Al}_{0.2} \mathrm{Ga}_{0.8}$ As $(0 \leq x \leq 0.09)$ MQW p-i-n structures grown by MBE to study the effect of compressive strain. $\operatorname{In}_{0.53 \pm x} \mathrm{Ga}_{0.47 \mp x} \mathrm{As} / \mathrm{InGaAsP} \mathrm{MQWs}$, grown by MOCVD, were used to measure the effect of compressive and tensile strain. The thickness of the MQW region is chosen such that it remains in the pseudomorphic regime. The effect of the strain should therefore be only on the well region of the MQW.
Ridge waveguides with contacts on the substrate and top $\mathrm{p}^{+}$laycrs were fabricated using photolithogra phy and ion-milling techniques. For modulation measurements $2-3 \mathrm{~mm}$ long waveguides were cleaved along the $[0 \overline{1}]$ and $[0 \overline{1} 1]$ crystallographic directions. The In$\mathrm{GaAs} / \mathrm{AlGaAs}$ diodes have leakage currents of $100 \mathrm{nA}$ at $-10 \mathrm{~V}$ and reverse breakdown voltages of $\sim 20 \mathrm{~V}$ in the dark. The InGaAs/InGaAsP diodes have a leakage currents of $100 \mu \mathrm{A}$ at $-4 \mathrm{~V}$ and reverse breakdown voltages of $\sim 6 \mathrm{~V}$ in the dark. The optical measurements were done with two sources: a Ti:Sapphire laser with $\lambda=$ $0.845-0.946 \mu \mathrm{m}$ for the InGaAs/AlGaAs system, and a Burleigh Color Center laser with $\lambda=1.48-1.67 \mu \mathrm{m}$ for the InGaAs/InGaAsP system. The laser light was endfired on to the cleaved edge of the waveguides through an objective lens. The near field pattern at the output of the waveguide was focused on to a silicon or germanium detector through an analyzer. For electroabsorption measurements, the output, with the analyzer adjusted for maximum transmission, was recorded for different applied bias for input polarizations both parallel (TE) and perpendicular (TM) to the layers. For phase modulation measurements the waveguide was excited with a polarization oriented at $45^{\circ}$ to the direction of the applied electric field to equally excite both TE and TM modes in the guide.

We have fixed the energy difference between the excitation and the excitonic peak to be $\sim 90 \mathrm{meV}$ and $15 \mathrm{meV}$ for the $\operatorname{In}_{x} \mathrm{Ga}_{1-x} \mathrm{As} / \mathrm{Al}_{0.2} \mathrm{Ga}_{0.8} \mathrm{As}$ and the $\mathrm{In}_{0.53 \pm x} \mathrm{Ga}_{0.47 \mp x} \mathrm{As} / \mathrm{InGaAsP}$ systems, respectively. By fixing the excitation distance, we can extract the effect of the strain on the electro-optic coefficients. The electrooptic coefficients $r_{63}$ and $\left(R_{33}-R_{13}\right)$ were obtained by comparing the measured phase changes with the calculated values according to ${ }^{3}$ :

$$
\Delta \underset{\substack{[110] \\[1 \overline{1} 0]}}{ }=\frac{\pi n_{1 \|}^{3} L}{\lambda}\left[ \pm \Gamma_{1} r_{63} \bar{E}_{j}+\Gamma_{2}\left(R_{33}-R_{13}\right) \bar{E}_{j}^{2}\right]+\Delta \Phi_{0}
$$

where $n_{\|}$is the average refractive index in the multilayer for polarization parallel to the layer, $E_{j}$ is the average junction electric field, $\Gamma_{1}$ and $\Gamma_{2}$ are the overlap factors of the optical mode with the junction electric field and the square of the junction electric field, respectively, and $L$ is the length of the waveguides. The built-in birefringence in the MQW, $\Delta \Phi_{0}$, can be expressed as:

$$
\Delta \Phi_{0}=\left(\beta_{T E}-\beta_{T M}\right) L=\Delta n L\left(\frac{2 \pi}{\lambda}\right)
$$

where

$$
\Delta n=\left(n_{\|}-n_{\perp}\right) .
$$

The values of $\Gamma_{1}$ and $\Gamma_{2}$ in our samples are 0.12 and 0.15 , respectively. Variations of $\Gamma_{1}$ and $\Gamma_{2}$ with wavelength are assumed to be small and the junction electric field is assumed to be constant. 


\section{Results and Discussion}

The values of the linear and quadratic electro-optic coefficients obtained by fitting the data to Eqn. 4 are given in Table 1 for the InGaAs/AlGaAs samples.

Table 1: Measured linear and quadratic electro-optic coefficients in $\mathrm{In}_{x} \mathrm{Ga}_{1-x} \mathrm{As} / \mathrm{Al}_{0.2} \mathrm{Ga}_{0.8}$ As strained MQW.

\begin{tabular}{ccccc}
\hline \hline$x$ & $\begin{array}{c}\lambda \\
{[\mu \mathrm{m}]}\end{array}$ & $\begin{array}{c}\Delta \mathrm{E}^{(\boldsymbol{a})} \\
{[\mathrm{meV}]}\end{array}$ & $\begin{array}{c}r_{63} \\
{\left[10^{-12} \mathrm{~m} / \mathrm{V}\right]}\end{array}$ & $\begin{array}{c}R_{33}-R_{13} \\
{\left[10^{-12} \mathrm{~m}^{2} / \mathrm{V}^{2}\right]}\end{array}$ \\
\cline { 2 - 2 } 0 & 0.8965 & 92 & 1.08 & 3.48 \\
0.03 & 0.9215 & 100 & 1.09 & 9.09 \\
0.06 & 0.9345 & 89 & 1.10 & 15.54 \\
0.09 & 0.9460 & 77 & 1.29 & 34.40 \\
\hline \hline
\end{tabular}

(a) Energy below exciton peak.

The values obtained for the InGaAs/InGaAsP system are listed in Table 2.

Table 2: Measured linear and quadratic electro-optic coefficients in $\mathrm{In}_{0.53+x} \mathrm{Ga}_{0.47-x} \mathrm{As} / \mathrm{InGaAsP}$ strained MQW.

\begin{tabular}{cccc}
\hline \hline$x$ & $\begin{array}{c}\lambda \\
{[\mu \mathrm{m}]}\end{array}$ & $\begin{array}{c}r_{63} \\
{\left[10^{-12} \mathrm{~m} / \mathrm{V}\right]}\end{array}$ & $\begin{array}{c}R_{33}-R_{13} \\
{\left[10^{-12} \mathrm{~m}^{2} / \mathrm{V}^{2}\right]}\end{array}$ \\
\hline-0.08 & 1.584 & 2.83 & 146 \\
0 & 1.65 & 3.74 & 282 \\
0.07 & 1.67 & 4.10 & 389 \\
\hline \hline
\end{tabular}

For both quantum well systems, the data indicates an increase in the electro-optic coefficients with the use of compressive strain. The major change, though, is manifested in the quadratic electro-optic coefficient $R_{33}-R_{13}$. In addition, the data for the $\operatorname{In}_{0.45} \mathrm{Ga}_{0.55} \mathrm{As} / \mathrm{InGaAsP}$ system shows a decrease in the electro-optic coefficients.

We have theoretically formulated the changes in the refractive index due to strain and find that our experimental results are in agreement with the calculated values. Figure 2 shows the experimentally measured data and the theoretically calculated results for the InGaAs/AlGaAs system. Figure 3 shows phase change as a function of applied field for the InGaAs/InGaAsP systems. We are currently calculating the changes in the refractive indices for the InGaAs/InGaAsP system and do not present them at this time.

\section{Conclusion}

We report the effect of strain on the refractive in$\operatorname{dex}$ of InGaAs/AlGaAs and InGaAs/InGaAsP MQWs. The experimental data show that it is possible to slightly

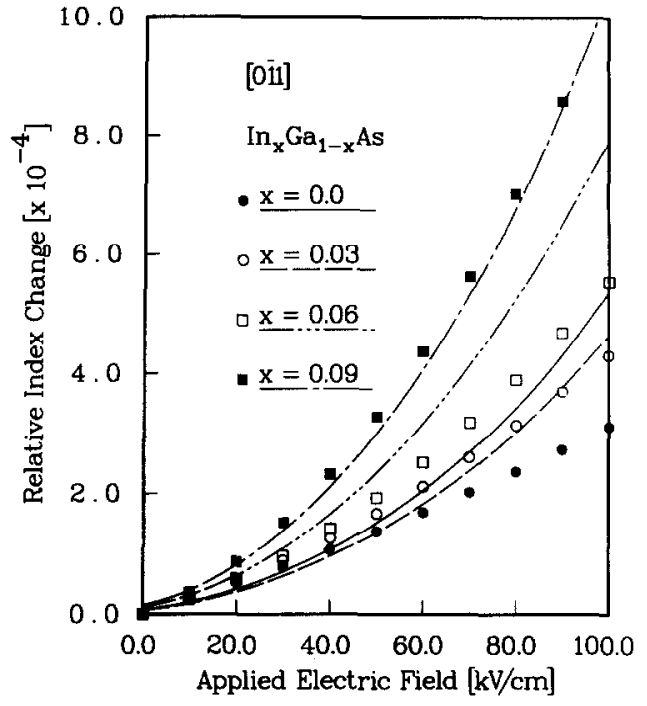

Figure 2:

Experimentally measured (points) and theoretically calculated (lines) values of $\Delta n$ as a function of applied electric field in the $\mathrm{In}_{x} \mathrm{Ga}_{1-x} \mathrm{As} / \mathrm{Al}_{0.20} \mathrm{Ga}_{0.80} \mathrm{As} \mathrm{MQW}$ system. The different curves are for varying amounts of strain in the well.

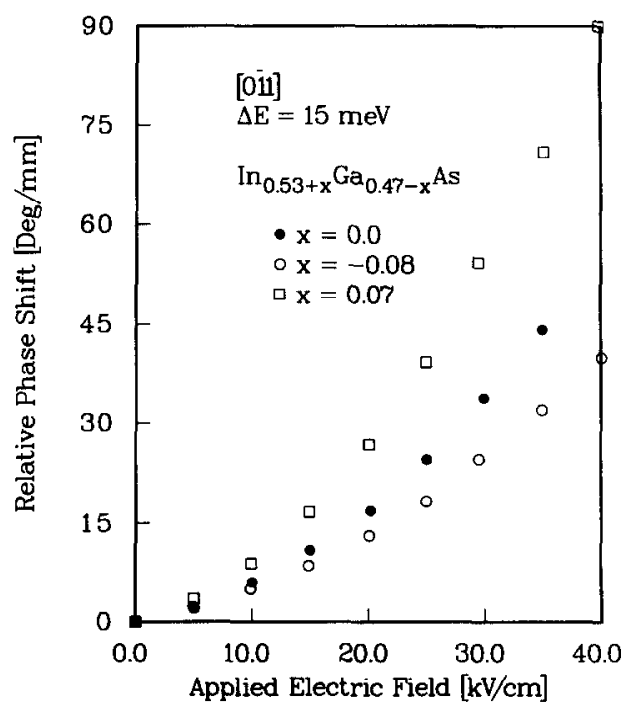

Figure 3:

Phase difference between TE- and TM-like modes for propagation in the $[0 \overline{1} 1]$ direction in the $\mathrm{In}_{0.53+x} \mathrm{Ga}_{0.47-x} \mathrm{As} / \mathrm{InGaAsP}$ system. 
tailor the linear electro-optic coefficient, $r_{63}$, while drastically changing the quadratic electro-optic coefficient, $R_{33}-R_{13}$, with the use of compressive and tensile strain. The theoretical formalism shows good agreement with the experimental data.

Acknowledgement - This work is supported by the Office of Naval Research under Grant N00014-90-J-1831 and the Army Research Office (URI Program) under contract DAAL03-87-K-0007. The authors gratefully acknowledge the help and facilities provided by S. Gupta and other menbers of the Ultrafast Sciences Laboratories, University of Michigan, for some of the measurements. One of us (MJL) wishes to acknowledge Bill Perez for expert assistance with epitaxial growth and characterization and John Turner for SIMS characterization.

\section{References}

1. H. Yamamoto, M Asada, and Y. Suematsu, Electronics Letters, 21, 579 (1985).

2. M. Glick, D. Pavuna, and F. K. Reinhart, Electronics Letters, 23, 1235 (1987).

3. U. Das, Y. Chen, P. K. Bhattacharya, and P. R. Berger, Applied Physics Letters, 53, 2129 (1988).

4. J. M. Luttinger and W. Kohn, Physical Review, 97, 869 (1955).

5. H. Kato, N. Iguchi, S. Chika, M. Nakayama and N. Sano, Journal of Applied Physics, $\underline{52}, 588$ (1986).

6. M. Jaffe and J. Singh, Journal of Applied Physics, $\underline{65}, 338$ (1989).

7. S. C. Hong, M. Jaffe and J. Singh, IEEE Journal of Quantum Electronics, 23, 2181 (1987).

8. J. P. Loehr and J. Singh, to be published in Physical Review B.

9. J. S. Weiner, D. A. B. Miller and D. S. Chemla, Applied Physics Letters, 50, 842 (1987). 